\title{
Papel do agente comunitário de saúde no controle do estoque domiciliar de medicamentos em comunidades atendidas pela estratégia de saúde da família
}

\author{
The role of the community health agent in control \\ of the in-house stock of medication in communities \\ served by the family health strategy
}

\author{
Gabriela Laste ${ }^{1}$ \\ Alícia Deitos ${ }^{2}$ \\ Carla Kauffmann ${ }^{2}$ \\ Luís César de Castro ${ }^{2}$ \\ Iraci Lucena da Silva Torres ${ }^{1}$ \\ Luciana Carvalho Fernandes ${ }^{2}$
}

${ }^{1}$ Departamento de Farmacologia, Programa de Pós-Graduação em Medicina, Ciências Médicas, Universidade Federal do Rio Grande do Sul. Rua Sarmento Leite, s/n. 90050170 Porto Alegre RS. gabrielalaste@gmail.com

${ }^{2}$ Centro de Ciências Biológicas e da Saúde, Centro Universitário Univates
Abstract The scope of this study was to investigate the in-house stock of medication in communities served by the Family Health Strategy (FHS) in a city located in the south of Brazil. A sample of 10\% of households in five FHS areas was studied. Data were collected by Community Healthcare Workers using the adapted Fernandes questionnaire (2000). The mean age of the interviewees was 42 years and of the residents was 16 years, 51\% of which were female. Low income (1 to 3 minimum wages) was observed (69.1\%), and low education levels (0 to 4 years of study) (42\%). Of the households visited 98.7\% had at least one medication in stock. In 533 of the storage places located, 43.5\% of such places were exposed to heat, $39.6 \%$ were exposed to humidity and $16.51 \%$ to light. Of the 2,717 medicines found, $6.9 \%$ were past the validity date, $35.3 \%$ were not in the secondary wrapping and $67.9 \%$ were without the patient information leaflet. Analgesics were the most common medications in in-house stock (21\%). This study revealed the need for a multi-professional team to promote health and the rationalization of in-house stock since the Community Healthcare Worker is an indispensable professional to promote the rational use of medication. Key words Rational use of medication, Family healthy strategy
Resumo $O$ objetivo deste estudo foi avaliar o estoque domiciliar de medicamentos em comunidades atendidas pela Estratégia de Saúde da Família (ESF) em município localizado no Sul do Brasil. Foram amostrados $10 \%$ dos domicílios de cinco ESF. Os dados foram coletados por Agentes Comunitários de Saúde (ACS) através de questionário adaptado. A idade média dos entrevistados foi de 42 anos e dos moradores foi de 16 anos, destes $51 \%$ eram mulheres. Foi observada renda de 1 a 3 salários mínimos (69,1\%), e escolaridade de 0 a 4 anos de estudo (42\%). Dos domicílios visitados, 98,7\% apresentavam pelo menos um medicamento estocado. Dos 533 lugares de guarda averiguados, $43,5 \%$ estava exposto ao calor, $39,6 \%$ a umidade, 16,5\% a luz. Dos 2.717 medicamentos encontrados, 6,9\% estavam vencidos, 35,3\% não estavam dentro da embalagem secundária, quando esta se aplica, 67,9\% não continham a presença de bula. Os fármacos analgésicos foram os mais prevalentes no estoque (21\%). Os resultados nos levam a concluir que há necessidade de equipe multiprofissional para a promoção de saúde e a racionalização dos estoques domiciliares de medicamentos, sendo que o ACS é um profissional indispensável para a promoção do uso racional de medicamentos.

Palavras-chave Farmácia domiciliar, Uso racional de medicamentos, Estratégia de saúde da família, Sistema Único de Saúde 


\section{Introdução}

A estratégia de saúde da família (ESF) está pautada, dentre outras diretrizes, no trabalho em equipe multidisciplinar ${ }^{1-4} \mathrm{e}$, entre os profissionais, o agente comunitário de saúde (ACS) é um dos responsáveis pelo desenvolvimento de atividades de prevenção de doenças e promoção da saúde por meio de ações educativas individuais e coletivas, nos domicílios e na comunidade ${ }^{5}$.

Este tipo de estratégia ultrapassa o cuidado individualizado, focado na doença. Ao trabalhar na família deve-se observar que a mesma é impulsionada por diferentes e conflitantes representações e motivações que perpassam, entre outros, os aspectos físicos (território), social (constituição de vizinhança) e relacional ${ }^{1,3,6}$. Sendo, portanto, de fundamental importância que a equipe de saúde conheça o perfil da comunidade na qual está inserida, a fim de que as propostas de promoção, recuperação e manutenção da saúde sejam mais diretas e eficientes ${ }^{1}$.

O SUS necessita superar alguns desafios, entre os quais, a busca do uso racional de medicamentos. Para tanto, o Ministério da Saúde tem se preocupado em transformar os ACS em fomentadores do uso racional de medicamentos em suas comunidades ${ }^{5}$. Esta afirmação pode ser evidenciada na publicação pelo Ministério da Saúde da cartilha "O trabalho dos ACS na promoção do uso correto de medicamentos".

Um dos aspectos relevantes para a promoção do uso racional de medicamentos é a observação da qualidade do produto que está sendo administrado, estando esta relacionada à manutenção da estabilidade do fármaco frente às condições de armazenamento e manuseio dos mesmos?

Nos estoques domiciliares pode haver medicamentos decorrentes de sobras de tratamentos anteriores, prescritos para tratamento de transtornos agudos e crônicos, ou por aqueles utilizados através da automedicação ${ }^{8}$. Armazenar medicamentos nos domicílios é uma prática comum, podendo representar um potencial risco para o surgimento de agravos à saúde. A farmácia domiciliar depositada em lugares inadequados pode propiciar consumo irracional e desperdício, incluindo a facilitação da automedicação não responsável, bem como o aumento do risco de exposições tóxicas não intencionais e intencionais?.

$\mathrm{O}$ armazenamento adequado e a preservação dos medicamentos são fundamentais para a sua adequada eficácia. Sendo assim, o objetivo deste estudo foi avaliar o estoque domiciliar de medicamentos em comunidades atendidas por ESF em município localizado Rio Grande do Sul.

\section{Métodos}

Realizou-se um estudo transversal populacional em áreas de abrangência de ESF em município localizado no Vale do Taquari, Rio Grande do Sul. A estimativa populacional do município, para 2009 , foi de $70.024^{10}$. O município é constituído por 6 ESF, por 6 Unidade Básica de Saúde (UBS), 3 Centros de Saúde e 1 Programa agentes comunitários de saúde (PACS). Além disso, conta com um profissional farmacêutico responsável por esses estabelecimentos de saúde existentes. A primeira ESF iniciou trabalho em 1999, sendo que em 2006 já estavam implantadas todas as existentes. Os critérios utilizados no município para a implementação de ESF são: indicadores de saúde, mortalidade infantil, condições socioeconômicas e epidemiológicas, acesso aos serviços de saúde e área de risco à saúde (condições sanitárias).

Amostragem foi composta de $10 \%$ dos domicílios de cada uma das áreas de abrangência das ESF. Cada agente comunitário é responsável por realizar visita domiciliar em um determinado número de famílias que residem em uma área territorial restrita. Dentro desta área as famílias são cadastradas, sendo que nos cadastros constam o endereço e dados relacionados à mesma. A numeração das famílias é realizada a partir da proximidade de localidade das ruas, portanto cada microárea corresponde a uma localidade (ruas próximas). Para se estabelecer quais os domicílios que seriam visitados, optou-se por um sorteio de $10 \%$ das famílias abrangidas por cada ACS a partir da enumeração das famílias já estabelecidas em cada microárea das ESF. Caso o número sorteado não contasse com uma família cadastrada, considerou-se o número seguinte a este. Desta forma, todas as microáreas foram amostradas.

A coleta de dados foi realizada no período de junho a setembro de 2009, empregando-se um questionário semiestruturado adaptado de Fernandes ${ }^{11}$. A coleta de dados foi realizada pelos ACS visando diminuir o desconforto do entrevistado ao responder questões de cunho pessoal e, consequentemente, aumentar a precisão das respostas, uma vez que entrevistador e entrevistado já se conhecem através das visitas domiciliares realizadas por estes profissionais.

Os ACS receberam treinamento prévio e foram monitorados durante toda a coleta de dados. Primeiramente, foi aplicado um questionário aos ACS, elaborado através do conteúdo da cartilha "O trabalho dos ACS na promoção do uso correto de medicamentos" ${ }^{\prime \prime}$, a partir das dificuldades visualizadas, foram abordados as- 
pectos relevantes relacionados aos medicamentos, tais como tipos (magistrais, oficinais, especialidade farmacêutica), vias de administração, formas farmacêuticas, entre outros. Além disso, foram treinados sobre a aplicação do instrumento de pesquisa, bem como sobre a forma de apresentação do projeto para o sujeito da pesquisa, e coleta da assinatura no Termo de Consentimento Livre e Esclarecido (TCLE). O projeto foi aprovado pelo Comitê de Ética em Pesquisa (COEP) do Centro Universitário Univates.

Foram incluídos na pesquisa os indivíduos com idade igual ou superior a 18 anos, que concordassem em assinar o TCLE e fossem responsáveis pela provisão de medicamentos na residência visitada. Foram excluídos os usuários com dificuldade de comunicação ou cognitiva.

Durante a entrevista foram compiladas informações sobre os moradores e domicílio, tais como idade, escolaridade e renda, número de cômodos da residência, número de moradores, ocupação do chefe da família. Além disso, foram analisados os móveis e locais de guarda dos medicamentos quanto à exposição à luz, calor e umidade; se estavam ou não ao alcance de crianças e se haviam crianças residentes no domicílio.

Com relação aos medicamentos, avaliou-se a forma de aquisição (mediante compra ou se fornecimento pela rede de saúde municipal, com ou sem prescrição médica), a data de validade no momento da entrevista, se estavam acondicionados dentro da embalagem secundária e primária, se constava bula, e se os mesmos ainda estavam em uso. Para identificação das classes terapêuticas de medicamentos que constavam nos estoques, estes foram classificados através do uso do sistema de classificação Anatomical Therapeutic Chemical (ATC) ${ }^{12}$, já os fitoterápicos e oficinais foram agrupados em separado.

O banco de dados foi construído no programa EPI-INFO (versão 3.3.2, 2005) e pelo SPSS (versão 8.0, 1998). O plano de análise dos dados incluiu análises descritivas pela média de distribuição da frequência das variáveis.

\section{Resultados e discussão}

Para se obter uma amostra representativa das ESF presentes no município, foi avaliado o estoque domiciliar de 473 residências de 5 das 6 ESF. Uma das ESF recusou sua participação por ter realizado trabalho similar sobre os estoques domiciliares de medicamentos com os ACS. A média de idade dos indivíduos entrevistados foi de 42 anos e a média dos moradores foi de 16 anos, com variação entre 0 a 83 anos. Destes, 51\% eram mulheres, sendo que a maioria $(42 \%)$ possuía 0 a 4 anos de estudo e 69,1\% apresentavam renda familiar de 1 a 3 salários mínimos.

O baixo nível de escolaridade e de renda pode ser considerado como barreira para o armazenamento racional de medicamentos, exigindo que os profissionais de saúde utilizem a linguagem adequada ao nível cultural do indivíduo, tornando, desta forma, a informação sobre a guarda de medicamentos acessível ao usuário.

Nas residências pesquisadas, entre as profissões encontradas, destacam-se: aposentados e pensionistas $(22,8 \%)$, serviços gerais $(13,5 \%)$ e trabalhadores da construção civil $(10,8 \%)$. O número de moradores por domicílio variou de 1 a 9, sendo que em sua maioria havia 3 a 4 moradores (51,6\%). Quanto ao tamanho das residências, $44,2 \%$ dos domicílios tinham 5 a 6 cômodos, 27,2\% mais de 6 cômodos, $20,5 \%$ de 3 a 4 cômodos (Tabela 1). Foi considerado cômodo cada parte do domicílio separada por paredes.

Tabela 1. Caracterização dos domicílios e famílias.

\begin{tabular}{lrr}
\hline \multicolumn{1}{c}{ Características } & No & \multicolumn{1}{c}{$\%$} \\
\hline Número de cômodos & & \\
1 - 2 cômodos & 14 & 2,9 \\
3 - 4 cômodos & 97 & 20,5 \\
5 - 6 cômodos & 209 & 44,2 \\
mais que 6 cômodos & 129 & 27,2 \\
Não respondeu & 24 & 5,1 \\
Total & $\mathbf{4 7 3}$ & $\mathbf{1 0 0 , 0}$ \\
Número de moradores & & \\
1 - 2 moradores & 161 & 34,0 \\
3 - 4 moradores & 244 & 51,6 \\
5 - 6 moradores & 62 & 13,1 \\
mais que 6 moradores & 6 & 1,3 \\
Total & $\mathbf{4 7 3}$ & $\mathbf{1 0 0 , 0}$ \\
Ocupação do chefe da família* & & \\
Aposentado/pensionista & 108 & 22,8 \\
Serviços gerais & 64 & 13,5 \\
Trabalhador da construção civil & 51 & 10,8 \\
Autônomo & 50 & 10,6 \\
Auxiliar produção indústria & 46 & 9,7 \\
Motorista & 23 & 4,9 \\
Mecânico & 20 & 4,2 \\
Do lar & 15 & 3,2 \\
Trabalhador do comércio & 13 & 2,8 \\
Outros & 57 & 12,0 \\
Não respondeu & 26 & 5,5 \\
Total & $\mathbf{4 7 3}$ & $\mathbf{1 0 0 , 0}$ \\
\hline * & &
\end{tabular}

* os dados de ocupação foram agrupados segundo a classificação fornecida pelo entrevistado. 
Das 473 entrevistas realizadas, em 467 domicílios $(98,7 \%)$ foi encontrado pelo menos um medicamento em estoque. Estudo de Dal Pizzol et al. ${ }^{8}$ demonstraram uma prevalência de $94 \%$ de estoque domiciliar concordando com Schenkel et al. ${ }^{13}$ que demonstraram $97 \%$ de prevalência.

O número de locais de guarda de medicamentos variou de 1 a 3 , sendo que a maioria das residências possuía apenas um $(89,2 \%)$, geralmente a cozinha. Estas características foram encontradas em outros estudos ${ }^{7,13,14}$. Dos 533 lugares de guarda averiguados, $43,5 \%$ estavam expostos ao calor, $39,6 \%$ à umidade, $16,5 \%$ à luz. Portanto, os usuários têm a percepção que os medicamentos precisam ser abrigados da luz, porém o mesmo não se verifica para umidade e calor. Com isso, reforça-se novamente a importância de que orientações fornecidas sejam adequadas à cultura do individuo.

Sobre a exposição dos medicamentos a crianças, em $42 \%$ dos domicílios havia medicamentos ao alcance de crianças, porém não havia crianças residindo no domicílio. Já em $37,3 \%$ das residências o estoque estava ao alcance de crianças e havia crianças residindo no domicílio (Tabela 2). Estudo transversal envolvendo as intoxicações medicamentosas agudas não-intencionais aten-

Tabela 2. Condições do estoque domiciliar de medicamentos.

\begin{tabular}{|c|c|c|}
\hline Condições & No & $\%$ \\
\hline \multicolumn{3}{|l|}{ Exposição ao calor } \\
\hline Sim & 232 & 43,5 \\
\hline Não & 301 & 56,5 \\
\hline Total $^{*}$ & 533 & 100,0 \\
\hline \multicolumn{3}{|l|}{ Exposição a crianças } \\
\hline $\begin{array}{l}\text { Está exposto e há crianças no } \\
\text { domicílio }\end{array}$ & 199 & 37,3 \\
\hline $\begin{array}{l}\text { Não está exposto a crianças e } \\
\text { há crianças no domicílio }\end{array}$ & 64 & 12,0 \\
\hline $\begin{array}{l}\text { Está exposto a crianças e não } \\
\text { há crianças no domicílio }\end{array}$ & 224 & 42,1 \\
\hline $\begin{array}{l}\text { Não está exposto a crianças e } \\
\text { não há crianças no domicílio }\end{array}$ & 46 & 8,6 \\
\hline Total $^{*}$ & 533 & 100,0 \\
\hline \multicolumn{3}{|l|}{ Exposição a luz } \\
\hline Sim & 88 & 16,5 \\
\hline Não & 445 & 83,5 \\
\hline Total $^{*}$ & 473 & 100,0 \\
\hline \multicolumn{3}{|l|}{ Exposição a umidade } \\
\hline Sim & 211 & 39,6 \\
\hline Não & 322 & 60,4 \\
\hline Total $^{*}$ & 473 & 100,0 \\
\hline
\end{tabular}

${ }^{*} \mathrm{n}=533$ móveis de guarda. didas e registradas pelo Centro de Controle de Intoxicações de Maringá no período de janeiro a dezembro 2004, demonstrou que a maioria dos pacientes intoxicados são do sexo masculino, de faixa etária entre 0 a 4 anos de idade ${ }^{14}$. Dados da SINITOX, referentes ao ano de 2007, apontaram os medicamentos como principais agentes de intoxicação em seres humanos. Cerca de $25 \%$ dos acidentes ocorrem com crianças menores de 5 anos $^{15}$. Portanto, o local escolhido para a guarda dos medicamentos é de extrema importância, a fim de evitar riscos de intoxicação, principalmente quando há crianças na residência.

A forma de aquisição dos medicamentos do estoque foi essencialmente por meio compra (51,7\%), seguido da aquisição na Unidade Básica de Saúde (UBS) (44,9\%) (Tabela 3).

Tabela 3. Perfil dos medicamentos estocados $(n=2717)$.

\begin{tabular}{|c|c|c|}
\hline Variáveis & No & $\%$ \\
\hline \multicolumn{3}{|l|}{ Origem } \\
\hline Farmácia & 1405 & 51,7 \\
\hline Unidade Básica de Saúde & 1221 & 44,9 \\
\hline Hospital & 8 & 0,3 \\
\hline Amigos/familiares/vizinhos & 18 & 0,7 \\
\hline "Mercadinho" & 16 & 0,6 \\
\hline Outros & 49 & 1,8 \\
\hline Total & 2717 & 100,0 \\
\hline \multicolumn{3}{|l|}{ Validade } \\
\hline Vencidos & 187 & 6,9 \\
\hline Válidos & 2459 & 90,5 \\
\hline Sem data de validade & 71 & 2,6 \\
\hline Total & 2717 & 100,0 \\
\hline \multicolumn{3}{|l|}{ Presença de embalagem secundária } \\
\hline Sim & 1757 & 64,7 \\
\hline Não & 960 & 35,3 \\
\hline Total & 2717 & 100,0 \\
\hline \multicolumn{3}{|l|}{ Presença de embalagem primária } \\
\hline Sim & 2632 & 96,9 \\
\hline Não & 85 & 3,1 \\
\hline Total & 2717 & 100,0 \\
\hline \multicolumn{3}{|l|}{ Medicamentos com bula } \\
\hline Sim & 873 & 32,1 \\
\hline Não & 1844 & 67,9 \\
\hline Total & 2717 & 100,0 \\
\hline \multicolumn{3}{|l|}{$\begin{array}{l}\text { Medicamentos com prescrição } \\
\text { médica }\end{array}$} \\
\hline Sim & 2162 & 79,6 \\
\hline Não & 555 & 20,4 \\
\hline Total & 2717 & 100,0 \\
\hline \multicolumn{3}{|l|}{ Medicamentos em uso } \\
\hline Sim & 1807 & 66,5 \\
\hline Não & 910 & 33,5 \\
\hline Total & 2717 & 100,0 \\
\hline
\end{tabular}


Apesar de o usuário residir em comunidade de abrangência de ESF, mesmo assim efetua a compra dos medicamentos. O que pode estar relacionado ao fato de que o medicamento não é apenas visualizado na sua dimensão terapêutica, mas, também como um bem de consumo. Esse tipo de fenômeno acontece, pois reflete o capitalismo, que transforma tudo em mercadoria com o objetivo de produzir lucros, e, neste contexto, a mercadoria medicamento é uma das materializações ou símbolos de saúde, um bem adquirível no mercado, e objeto de um processo social de produção de significado engendrado por uma dada formação social concreta, ou seja, como um símbolo ${ }^{7,16,17}$.

Dos 2.717 medicamentos encontrados, 90,5\% estavam válidos, $6,9 \%$ vencidos e $2,6 \%$ não apresentavam a informação sobre a data de validade. Como nosso estudo foi realizado em área de abrangência de ESF, os ACS têm como rotina realizar o recolhimento dos produtos vencidos nos domicílios, o que explica o baixo número de medicamentos nestas condições encontrados em relação a outros estudos ${ }^{13,18}$, em que foram encontrados $16 \%$ e $20,5 \%$ respectivamente.

Quanto ao invólucro primário, o qual compreendeu o medicamento acondicionado em blister, 33,3\% dos medicamentos não continham blister, eram pomadas, soluções entre outros e, $66,7 \%$ estavam acondicionados em blisters (comprimidos) e, destes, apenas 4,7\% estavam armazenados fora dos mesmos (Tabela 3). Quanto à presença de embalagem secundária, 35,3\% dos medicamentos estocados não estavam na embalagem e em $67,9 \%$ dos medicamentos não havia a presença da bula (Tabela 3 ). Sabe-se que na rede pública há distribuição de medicamentos sem embalagem secundária e bula, o que acaba contribuindo para um acondicionamento inadequado dos fármacos ${ }^{19}$. Soma-se a isto, o fato de que muitos medicamentos possuem embalagem primária semelhante o que pode gerar erros na administração do produto. No Brasil, a bula ainda representa o principal material informativo referente aos medicamentos produzidos pela indústria farmacêutica e há obrigatoriedade da sua inclusão na embalagem dos medicamentos ${ }^{20}$.

A maioria dos medicamentos do estoque foi adquirida sob prescrição médica (79,6\%). A elevada percentagem de medicamentos fornecidos com prescrição pode estar relacionada ao fato da amostra ser de área de abrangência de ESF, sendo assim, os indivíduos retiram os medicamentos na UBS mediante prescrição e, quando não encontram o medicamento disponível, pro- videnciam a compra. Quanto à situação do estoque, $66,5 \%$ dos medicamentos estavam ainda em uso. Esse percentual reflete um estoque ativo, típico de famílias que possuem sujeitos em tratamento para doenças crônicas (Tabela 3).

$\mathrm{O}$ número de medicamentos estocados por residência variou de 1 a 21 unidades, com média de 5,75 medicamentos, desvio padrão $\pm 3,79$. Sendo que, dos 2.717 medicamentos estocados 95,5\% eram especialidades farmacêuticas, $1,7 \%$ produtos oficinais e 1,9\% fitoterápicos. Este número de medicamentos estocados foi inferior ao obtido em outros estudos similares ${ }^{8,13}$ que demonstraram média de 12 e 20 medicamentos estocados respectivamente. Cabe ressaltar que aqueles não se restringiram a populações de abrangência de ESF.

Analisando por classe terapêutica, os fármacos analgésicos e antipiréticos foram os mais prevalentes no estoque (21\%), seguido dos antiinflamatórios e antirreumáticos (10,2\%) (Tabela 4). Nos estoques domiciliares, observou-se que paracetamol $(50,8 \%)$ foi o mais frequente dos analgésicos e o ibuprofeno $(59,9 \%)$ entre os antiinflamatórios.

Entre os fármacos com ação no sistema cardiovascular, destacaram-se os que atuam no sistema renina angiotensina $(5,9 \%)$ e diuréticos $(5,3 \%)$. Outros fármacos como anti-histamíni$\cos (4,9 \%)$, antibacterianos $(4,5 \%)$, antiácidos, antiulcerosos e anti-inflamatórios (3,7\%) também foram encontrados com frequência nos estoques domiciliares (Tabela 4 ).

Os medicamentos mais prevalentes são aqueles de fácil acesso, tais como analgésicos e antiinflamatórios, adquiridos sem receita médica e utilizados na forma de automedicação ${ }^{19}$. Corroborando resultados de Lima et al. ${ }^{19}$ que analisaram o armazenamento de medicamentos em domicílios visitados por agentes comunitários de saúde e enfermeiro, constatando grande percentual de medicamentos estocados vendidos sem receita médica. Brum et al. ${ }^{18}$ analisaram o estoque domiciliar de medicamentos através de campanha de arrecadação e obtiveram as classes terapêuticas semelhantes, porém as mais prevalentes foram as dos anti-hipertensivos e antidiabéticos, demonstrando possível não adesão ao tratamento. Cabe ressaltar que apesar da considerável prevalência desses fármacos encontrados em nosso estudo, não foi avaliado quais destas classes terapêuticas não estavam mais em uso.

Deve-se levar em conta que a hipertensão arterial possui elevada prevalência em nosso meio, atingindo entre $22,3 \%$ a 43,9\% da população adul$\mathrm{ta}^{21}$. Além disso, o Programa Nacional de Assis- 
Tabela 4. Medicamentos estocados nos domicílios conforme classe terapêutica.

\begin{tabular}{|c|c|c|}
\hline Classe terapêutica $^{*}$ & No & $\%$ \\
\hline \multicolumn{3}{|l|}{ Trato alimentar e metabolismo } \\
\hline $\begin{array}{l}\text { Antiácidos, antiulcerosos e anti- } \\
\text { inflamatórios }\end{array}$ & 101 & 3,7 \\
\hline Antiespasmódicos, anticolinérgicos e & 89 & 3,3 \\
\hline propulsivos & 56 & 2,1 \\
\hline \multicolumn{3}{|l|}{ Antidiabéticos } \\
\hline \multicolumn{3}{|l|}{ Sangue e órgãos formadores de sangue } \\
\hline Preparações antianêmicas & 21 & 0,8 \\
\hline \multicolumn{3}{|l|}{ Sistema cardiovascular } \\
\hline Diuréticos & 145 & 5,3 \\
\hline Agentes betabloqueadores & 74 & 2,7 \\
\hline $\begin{array}{l}\text { Fármacos que atuam no sistema } \\
\text { renina angiotensina }\end{array}$ & 159 & 5,9 \\
\hline $\begin{array}{l}\text { Fármacos redutores dos lipídios } \\
\text { séricos }\end{array}$ & 45 & 1,7 \\
\hline \multicolumn{3}{|l|}{ Agentes dermatológicos } \\
\hline Antifúngicos & 39 & 1,4 \\
\hline Antibióticos e quimioterápicos & 29 & 1,1 \\
\hline Corticóides & 35 & 1,3 \\
\hline \multicolumn{3}{|l|}{ Sistema geniturinário e sexuais } \\
\hline $\begin{array}{l}\text { Anticoncepcionais para uso } \\
\text { sistêmico }\end{array}$ & 40 & 1,5 \\
\hline \multicolumn{3}{|l|}{ Antiinfecciosos de uso sistêmico } \\
\hline Antibacterianos & 123 & 4,5 \\
\hline \multicolumn{3}{|l|}{ Sistema músculo esquelético } \\
\hline $\begin{array}{l}\text { Anti-inflamatórios e } \\
\text { antirreumáticos }\end{array}$ & 277 & 10,2 \\
\hline \multicolumn{3}{|l|}{ Sistema nervoso } \\
\hline Analgésicos e outros antipiréticos & 571 & 21,0 \\
\hline Antiepilépticos & 40 & 1,5 \\
\hline Ansiolíticos & 45 & 1,6 \\
\hline Antidepressivos & 86 & 3,2 \\
\hline \multicolumn{3}{|l|}{ Preparações de uso nasal } \\
\hline Preparações nasais de uso tópico & 28 & 1,0 \\
\hline $\begin{array}{l}\text { Agentes desobstrutivos das vias } \\
\text { respiratórias }\end{array}$ & 42 & 1,5 \\
\hline Anti-histamínicos & 133 & 4,9 \\
\hline \multicolumn{3}{|l|}{ Fármacos diversos } \\
\hline Fármacos diversos & 539 & 19,8 \\
\hline Total & 2717 & 100,0 \\
\hline
\end{tabular}

*Segundo Classificação ATC, 2009.

\section{Considerações finais}

Este estudo demonstrou alta prevalência no consumo de analgésicos, obtidos sem apresentação da prescrição, podendo contribuir para a prática de automedicação, consequentemente aumentando o risco de intoxicação medicamentosa. Um exemplo que chama a atenção é o uso de paracetamol, que em doses não terapêuticas pode provocar hepatoxicidade ${ }^{23}$. Além disso, foram apresentados alguns problemas nos estoques domiciliares de medicamentos, tais como armazenamento inadequado dos mesmos e a necessidade dos ACS orientarem de forma clara ao usuário os locais de guarda dos medicamentos, principalmente no que se refere ao calor, luz e umidade.

A maioria das ESF do município em estudo é constituída pela equipe multiprofissional básica, ou seja, com enfermeiro, médico, dentista, auxiliar/técnico de enfermagem. A ausência do farmacêutico nesta equipe leva os enfermeiros ou técnicos de enfermagem a terem que suprir a necessidade de informação dos pacientes sobre os medicamentos prescritos.

Nesse sentido, a criação dos Núcleos de Apoio à Saúde da Família constitui um passo importante para a consolidação da ESF e especialmente para o desenvolvimento e aprimoramento de um novo modelo de exercício do trabalho em equipe multiprofissional. Uma equipe de profissionais de diversas áreas, entre eles o farmacêutico, pode através do apoio matricial, auxiliar na tarefa de desenvolver atividades de promoção de saúde, como estratégias de racionalização dos estoques domiciliares de medicamentos e o recolhimento dos mesmos fora de uso e/ou fora do prazo de validade.

$\mathrm{Na}$ concepção de rede e apoio matricial ${ }^{24}$, os ACS têm papel fundamental, uma vez que estes trabalhadores dentro da equipe de referência são os que efetivamente se responsabilizam por entrar na casa do usuário e verificar a condição do estoque domiciliar de medicamentos. Desta forma, são suporte para a equipe na detecção de problemas potenciais e reais relacionados à farmacoterapia. tência Farmacêutica para Hipertensão Arterial e Diabetes Mellitus, denominado de Hiperdia, assegura o fornecimento dos medicamentos selecionados para o tratamento dessas patologias ${ }^{22}$, consequentemente, aumentando o acesso da população a esses medicamentos e, portanto, maior prevalência desses nos estoques domiciliares. 


\section{Colaboradores}

G Laste participou da elaboração do projeto de pesquisa, coleta de dados, análise de dados, confecção do artigo; A Deitos participou na confecção do artigo; C Kauffmann participou na elaboração do projeto de pesquisa; ILS Torres e LC Castro auxiliaram na confecção do artigo; LC Fernandes participou na elaboração do projeto de pesquisa, orientando a aluna no Trabalho de Conclusão de Curso, confecção do artigo.

\section{Referências}

1. Goulart BNG, Algayer AR. Características de um grupo de usuários do Programa Saúde da Família na cidade de Campo Bom (RS), Brasil em 2006. Cien Saude Colet 2007; 14(Supl.1):1379-1384.

2. Crevelim MA. Participação da Comunidade na equipe de Saúde da Família: é possível estabelecer um projeto comum entre trabalhadores e usuários? Ciência e Saúde 2005; 10(2):323-331.

3. Bertussi DC, Oliveira MSM, Lima JVC. A Unidade Básica de Saúde no contexto do Sistema de Saúde. In: Andrade SM, Santos DA, Cordoni Junior L, organizadores. Bases da Saúde Coletiva: Londrina: UEL; 2001. p. 133-144.

4. Carnelosso ML, Barbosa MA, Souza ALL, Monego ET, Carvalho MM. Enfermidades não transmissíveis na Atenção Básica: novo desafio para o PSF. Projeto de Desenvolvimento de Sistemas e Serviços de Saúde. Brasília: Organização Pan-americana de Saúde (OPAS), Ministério da Saúde; 2004. p 117-126.

5. Brasil. Ministério da Saúde (MS). O trabalho dos Agentes Comunitários de Saúde na promoção do uso correto de medicamentos. 2a ed. Brasília: Editora MS; 2006.

6. Ribeiro RM. As várias abordagens da Família no Cenário da Programa/Estratégia de Saúde da Família (PSF). Rev. Latino am enfermagem 2004; 12(4):658664.

7. Lima GB, Nunes LC, Barros JAC. Uso de Medicamentos armazenados em domicílio em uma população atendida pelo PSF. Cien Saude Colet 2010; 15(Supl. 3):3517-3522.

8. Dal Pizol TS, Piccoli A, Brugnera Q, Schenkel EP, Mengue SS. Análise dos Estoques Domiciliares de Medicamentos Essenciais no Sul do Brasil. Acta Farm. Bonaerense 2006; 25(4):601-607.

9. Tourinho FSV, Bucaretchi F, Stephan C, Cordeiro R. Farmácias domiciliares e sua relação com a automedicação em crianças e adolescentes. Jornal de Pediatria 2008; 84(5):416-422.

10. Banco de dados regional (BDR). 2009. [site na Internet]. [acessado 2009 nov 5]. Disponível em: http:/ /www.bdr.univates.br/

11. Fernandes LC. Caracterização e análise da farmácia caseira ou estoque domiciliar de medicamentos [dissertação]. Porto Alegre (RS): Universidade Federal do Rio Grande do Sul; 2000.

12. World Health Organization (WHO). WHO Collaborating Centre for Drug Statistics Methodology. ATC/DDD Index 2009. [site na Internet]. [acessado 2009 nov 5]. Disponível em: http://www.whocc.no/ atcddd/

13. Schenkel EP, Fernandes LC, Mengue SS. Como são armazenados os medicamentos nos domicílios? Acta Farm. Bonaerense 2005; 24(2):266-270.

14. Margonato FB, Thomson Z, Paoliello MMB. Determinantes nas intoxicações medicamentosas agudas na zona urbana de um município do Sul do Brasil. Cad Saude Publica 2008; 24(2):333-341.

15. Sistema Nacional de Informações Tóxico-Farmacológicas. Envenenamento doméstico, 2007. [site da Internet]. [acessado 2009 nov 20]. Disponível em: http://www.fiocruz.br/sinitox 
16. Pereira SLG. Os Investimentos das Indústrias Farmacêuticas Brasileiras em Programas Sociais: uma análise dos indicadores sociais de 2006. Saúde Soc. 2009; 18(1):19-28.

17. Lefévre F. O medicamento como mercadoria simbólica. São Paulo: Cortez; 1991.

18. Brum CA, Depizzol MCA, Lopes TV, Loures GF, Valadão AF. Avaliação do estoque de medicamentos das residências da Região do Vale do Aço MG. Rev. Bras. Farm 2007; 88(4):173-176.

19. Lima, GB, Araújo, EJF, Souza, KMH, Benvido, RF, Silva, WCS, Correa, RAC, Nunes, LCC. Avaliação da utilização de medicamentos armazenados em domicílios por uma população atendida pelo PSF. Rev. Bras. Farm 2008; 89(2):146-149.

20. Brasil. Portaria no 110, de 10 de março de1997. Diário Oficial da União 1997; 18 mar.

21. Brasil. Ministério da Saúde. Secretaria de Atenção à Saúde. Departamento de Atenção Básica. Hipertensão arterial sistêmica para o Sistema Único de Saúde. Brasília: Ministério da Saúde - Cadernos de Atenção Básica, n.16, série A. Normas e Manuais Técnicos; 2006 .

22. Brasil. Portaria Conjunta no 02, de 05 de março de 2002. Implantação do Plano de Reorganização da Atenção à Hipertensão Arterial e ao Diabetes Mellitus. Diário Oficial da União 2002; 20 mar.

23. Mitchel SJ, Hilmer SN, Matthews S. Hepatotoxicity of therapeutic short-course paracetamol in hospital inpatients: impact of ageing and frailty. J Clin Pharm Ther 2011; 36(3):327-335.

24. Campos GWS, Domitti AC. Apoio matricial e equipe de referência: uma metodologia para gestão do trabalho interdisciplinar em saúde. Cad Saude Publica 2007; 23(2):399-407.

Artigo apresentado em 15/05/2011

Aprovado em 18/04/2011

Versão final apresentada em 11/07/2011 